\title{
Methyl-CpG Binding Domain Protein 2 Inhibits the Malignant Characteristic of Lung Adenocarcinoma through the Epigenetic Modulation of 10 to 11 Translocation 1 and miR-200s
}

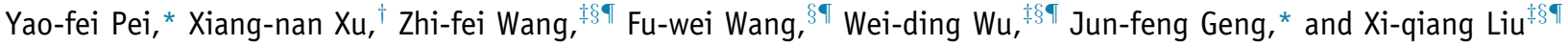

\begin{abstract}
From the Department of Thoracic Surgery, * Shanghai Chest Hospital, Shanghai Jiaotong University, Shanghai; the Department of Thoracic Surgery, ${ }^{\dagger}$ Shanghai General Hospital, Shanghai Jiaotong University School of Medicine, Shanghai; the Department of Hepatopancreatobiliary Surgery and Minimally Invasive Surgery, ${ }^{\ddagger}$ Zhejiang Provincial People’s Hospital, People’s Hospital of Hangzhou Medical College, Hangzhou; the Key Laboratory of Tumor Molecular Diagnosis and Individualized Medicine of Zhejiang Province ${ }^{\S}$, Hangzhou; and the Key Laboratory of Gastroenterology of Zhejiang Province Hangzhou, People's Republic of China
\end{abstract}

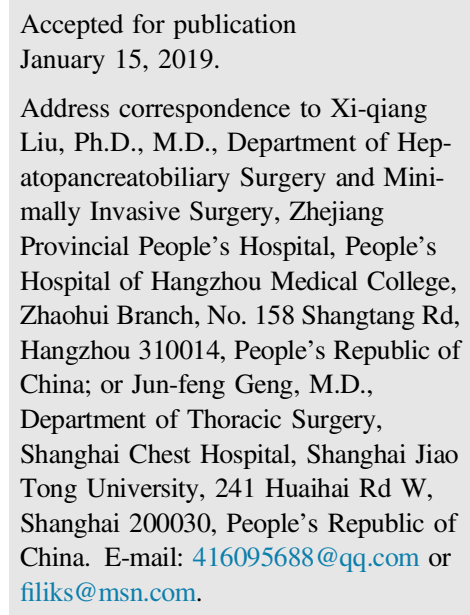

\begin{abstract}
It has been reported that disorders of epigenetic modulation play a critical role in carcinogenesis. Methyl- $\mathrm{CpG}$ binding domain protein 2 (MBD2) is known to act as an epigenetic modulator in various types of tumors; however, the role of MBD2 in lung adenocarcinoma (LUAD) remains unclear. Herein, we demonstrated the down-regulation of MBD2 in LUAD compared with adjacent nontumor tissues. The down-regulation of MBD2 in LUAD was correlated with metastasis and poor survival. In addition, MBD2 inhibited tumor metastasis by maintaining the expression of the miR-200s, which suppressed the invasive properties of tumors. Also, MBD2 positively correlated with 5-hydroxymethylcytosine content in the promoter of miR-200s. The conventional view is that MBD2 acts as a transcriptional suppressor. However, the data revealed that MBD2 may act as a transcriptional activator by recruiting 10 to 11 translocation 1 (TET1) and forming a chromatin-remodeling complex. The MBD2-TET1 complex locates to the TET1 promoter and removes the methyl residues in this region, thereby activating TET1 transcription. TET1 also acted as a tumor suppressor in LUAD. Taken together, the data demonstrate the correlation between MBD2, miR-200s, and TET1, and tumor suppressive effect of MBD2 through up-regulation of TET1 and the miR-200s. (Am J Pathol 2019, 189: 1065-1076; https://doi.org/ 10.1016/j.ajpath.2019.01.010)
\end{abstract}

Lung adenocarcinoma (LUAD) has become one of the most lethal tumors worldwide. ${ }^{1}$ Although there have been increasing advancements in treatments of surgery and chemotherapy, the prognosis of LUAD patients remains poor because of recurrence and remote metastasis. ${ }^{2}$ Aberrant epigenetic modulation always occurs in malignant tumors, which promotes aggressive characteristics in the tumors. ${ }^{3}$ Thus, it is of impending necessity to investigate the mechanism underlying the epigenetic modulation in LUAD.

Methyl-CpG binding domain protein 2 (MBD2) is a protein that binds $\mathrm{CpG}$ islands (CGIs) in promoters, which are the

\footnotetext{
Supported by the Natural Science Foundation of Zhejiang Province (General Project) grant LY18H160038 (X.-q.L), Projects of Medical and Health Technology Program of ZheJiang Province grant 2017KY223 (X.-q.L), the Launching Fund of Excellent Youth Scientific Research in Zhejiang People's Hospital grant ZRY2016B003 (X.-q.L), the National Key Research and Development Project in China grant 2018YFB1107104 (Z.-f.W.), Major Science and Technology Projects of Zhejiang Province grant 2017 C01020 (Z.-f.W.), the Public Welfare Project of Zhejiang Science and Technology Department grant 2015C33112 (Z.-f.W.), Zhejiang Provincial Health Department General Project grant 201477869 (Z.-f.W.), and Zhejiang Province Medical and Health Platform Key Project grant 201348036 (Z.-f.W.).

Y.-f.P. and X-n.X. contributed equally to this work.

Disclosures: None declared.
} 
regions where the DNA is methylated. ${ }^{4}$ Usually, MBD2 acts as a transcriptional repressor, silencing gene expression by recruiting histone deacetylases and DNA methyltransferases. ${ }^{5,6}$ However, certain studies have also reported that MBD2 acts as a transcriptional activator in cAMP-responsive promoters. $^{7}$ These controversial results regarding MBD2 indicate that MBD2 plays different roles based on the cell type. Aberrantly expressed MBD2 is always involved in many types of tumors, such as hepatocellular carcinoma, prostate cancer, and breast cancer. ${ }^{8}$ Devailly et $\mathrm{al}^{9}$ reported that MBD2 is profoundly involved in the oncogenic transformation of human mammary cells. Yuan et $\mathrm{al}^{10}$ reported that MBD2 promotes tumor growth and metastasis in colorectal cancer in association with histone deacetylase (HDAC) 1 . In the present study, it was determined that the down-regulation of MBD2 activates the epithelial-mesenchymal transition (EMT) and promotes tumor metastasis in LUAD.

miRNAs are noncoding small RNAs that target the $3^{\prime}$-untranslated region of mRNAs. The aberrant expression of miRNAs has been recognized as an initiator of the malignant transformation of cells. ${ }^{11}$ Furthermore, the miR-200s have been identified as tumor suppressors in many types of tumors. ${ }^{12}$ In addition, many researchers have reported that the miR-200s act as suppressors of the invasive properties of tumors. Shelton et $\mathrm{al}^{13}$ determined that the miR-200s inhibit liver metastasis in colorectal cancer and inhibit the EMT process by targeting zinc finger E-box binding homeobox (ZEB) 1 and ZEB2. Although the role of miR-200s has been extensively studied in various types of malignant tumors, there are few studies on the epigenetic modulation of the miR-200s. In this study, a positive correlation was demonstrated between the miR-200s and MBD2. Further investigation revealed the positive correlation between MBD2 and the 5-hydroxymethylcytosine (5-hmC) content in the CGIs upstream of the miR-200s.

The 10 to 11 translocation 1 (TET1), which is a member of the TET family, is implicated in converting 5-methylcytosine (5-mC) to 5 -hmC. ${ }^{14}$ The increase in 5 -hmC mediated by TET1 results in the demethylation of CGIs in specific gene promoters and, consequently, the activation of gene expression. ${ }^{15}$ Furthermore, TET1 often acts as a tumor suppressor, and its absence induces aggressive characteristics in malignant tumors, such as colon cancer, hepatic cancer, and lung cancer. ${ }^{16-18}$ It has been reported that depressed TET1 expression promotes cell migration and tumor metastasis. ${ }^{19}$ Chuang et al ${ }^{20}$ reported that TET1 suppresses tumor metastasis through the epigenetic modulation of invasive-suppressing miRNAs in hepatoma. A similar mechanism was also discovered in LUAD in the present study. Sun et $\mathrm{al}^{21}$ found that TET1 is regulated through the methylation status of CGIs in its own promoter. In this study, we demonstrated that MBD2 interacts with TET1 and forms a complex. This complex is located at the TETI promoter, where it acts as a transcriptional activator and increases TET1 transcription. Overall, the study shows the positive correlation between MBD2, miR-200s, and TET1 in LUAD, and suggests that TET1 is involved in an autoregulation that is mediated by MBD2.

\section{Materials and Methods}

\section{Tissues and Database}

The microarray data of LUAD patients were obtained from the Gene Expression Omnibus (GEO) database (https:// www.ncbi.nlm.nih.gov/geo; accession numbers GSE7670, GSE10072, GSE19188, GSE31210, GSE32863, and GSE8322). A total of 50 pairs of tumor and adjacent nontumor tissues were collected in cohort 1 and used for real-time quantitative PCR (qPCR). Cohort 2 contained 100 LUAD tumor samples, which were used for correlation analysis and methylation analysis. In addition, 150 formaldehyde-fixed and paraffin-embedded LUAD samples were collected in cohort 3 and used for the analysis of clinicopathological data. All samples were collected from LUAD patients who underwent surgery in Zhejiang Provincial People's Hospital (Hangzhou, China). Written informed consents were obtained from all patients. The present study was approved by the Ethics Committee of Zhejiang Provincial People's Hospital.

\section{Cell Culture}

Human LUAD cell lines (HCC827, PC9, H1650, H3255, H1975, and A549) were obtained from ATCC (Manassas, VA). These cell lines were cultured at $37^{\circ} \mathrm{C}$ with $5 \% \mathrm{CO}_{2}$. Culture medium was RPMI 1640 containing $10 \%$ fetal bovine serum.

\section{Cell Migration}

A cell migration assay was performed, as previously described. $^{22}$ The number of cells was determined using a microscope. All experiments were performed in triplicate.

\section{RNA Extraction and $q P C R$}

RNA extraction and qPCR were performed, as previously described. ${ }^{22}$ Glyceraldehyde-3-phosphate dehydrogenase and U6 were used as endogenous controls for mRNA and miRNA detection, respectively. All primers used in the study are listed in Table 1. All experiments were performed in triplicate.

\section{Detection of 5-mC and 5-hmC Content}

Detection of 5-mC and 5-hmC was performed using an EpiMark 5-hmC and 5-mC analysis kit (New England Biolabs, Ipswich, MA). Genomic DNA was incubated with T4 phage b-glucosyltransferase, according to the manufacturer's instructions. Then, the DNA was digested with the HpaII and MspI enzymes. Afterward, the digested products were amplified by PCR. The primers used are listed in Table 1. The 5-mC and 5-hmC content was calculated according to a previously described method. ${ }^{23}$ 
Table 1 List of Primers Used in This Study

\begin{tabular}{|c|c|}
\hline Primer name & Primer sequence \\
\hline \multicolumn{2}{|c|}{ Sequences of the primer pairs used for $q P C R$} \\
\hline U6 & $5^{\prime}$-CTCGCTTCGGCAGCACA-3' \\
\hline GAPDH & Reverse: 5'-AAGTGGTCGTTGAGGGCAATG-3' \\
\hline \multirow[t]{2}{*}{ TET1 } & Forward: 5'-CGCTACGAAGCACCTCTCTTA-3' \\
\hline & Reverse: 5'-CTTGCATTGGAACCGAATCATTT-3' \\
\hline $\operatorname{miR}-200 a$ & 5'-CATCTTACCGGACAGTGCTGGA-3' \\
\hline $\mathrm{miR}-200 \mathrm{~b}$ & 5'-CATCTTACTGGGCAGCATTGGA-3' \\
\hline $\operatorname{miR}-200 \mathrm{c}$ & $5^{\prime}$-CATCTTACTGGGCAGCAGCATTGGA-3' \\
\hline $\operatorname{miR}-126$ & 5'-CATTATTACTTTTGGTACGCG-3' \\
\hline $\operatorname{miR}-148$ & 5'-AAAGTTCTGAGACACTCCGACT-3' \\
\hline \multicolumn{2}{|c|}{ Sequences of the primer pairs used for methylated- and unmethylated-specific qPCR } \\
\hline \multirow[t]{2}{*}{ Methylated-specific miR-200a/b } & Forward: 5'-AGATTGGTTAGCGGGAGTTC-3' \\
\hline & Reverse: 5'-ATTTCGAAAACGACGAAACA-3' \\
\hline \multirow[t]{2}{*}{ Unmethylated-specific miR-200a/b } & Forward: 5'-GGAGATTGGTTAGTGGGAGTTT-3' \\
\hline & Reverse: 5'-AAATTTCAAAAACAACAAAACA-3' \\
\hline \multirow[t]{2}{*}{ Methylated-specific miR-200c } & Forward: 5'-TTCGTTGTTTGTTTTTCGAC-3' \\
\hline & Reverse: $5^{\prime}-$ CTCGTCCATACGCACTACAT-3' \\
\hline \multirow[t]{2}{*}{ Unmethylated-specific miR-200c } & Forward: 5'-GGGTTTGTTGTTTGTTTTTTGAT-3' \\
\hline & Reverse: 5'-ААССТСАТССАТАСАСАСТАСАТ-3' \\
\hline Methylated-specific & Forward: 5'-TTATGTAGTTTTATTTGTTTTTTTATTGTG-3' \\
\hline TET1 & Reverse: 5'-CAАСТCСАААССТАСАССАAC-3' \\
\hline TET1 & Reverse: $5^{\prime}$-CGCACCTCCCACCCTCGGGGCC-3' \\
\hline \multicolumn{2}{|c|}{ Sequences of the primer pairs used for BSP } \\
\hline \multirow[t]{2}{*}{ miR-200a/b } & Forward: 5'-GGTTTATGGGAGTTTAGGGGATATAT-3' \\
\hline & 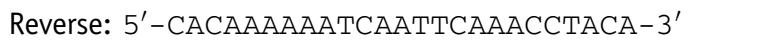 \\
\hline \multirow[t]{2}{*}{$\operatorname{miR}-200 c$} & Forward: 5'-GTATAGGTTGTTGGTTTGGGGTAG-3' \\
\hline & Reverse: 5'-ААТАAСССААТАААATСССАААТСТ-3' \\
\hline \multicolumn{2}{|c|}{ Sequences of the primer pairs used for ChIP-PCR } \\
\hline \multirow[t]{2}{*}{ TET1 } & Forward: 5'-TTTGGGAACCGACTCCTCACCT-3' \\
\hline & Reverse: 5'-TCGGGCAAACTTTCCAACTCGC-3' \\
\hline
\end{tabular}

BSP, bisulfite-sequencing PCR; ChIP, chromatin immunoprecipitation; GAPDH, glyceraldehyde-3-phosphate dehydrogenase; GlucMS, glucosylation of genomic DNA followed by methylation-sensitive; $\mathrm{PPCR}$, real-time quantitative PCR.

\section{Bisulfate Sequencing}

Genomic DNA was extracted and treated according to the guidelines of the Epi kit (Qiagen, Hilden, Germany). Then, the bisulfate-treated DNA was amplified with PCR (Table 1). Afterward, the PCR products were subcloned into the vector. Subsequently, 10 clones of each group were randomly chosen and sent for sequencing. The sequencing results were analyzed using the online tool QUMA (http://quma.cdb. riken.jp, last accessed December 2, 2018).

\section{ChIP}

A chromatin immunoprecipitation (ChIP) assay was performed, as previously described. ${ }^{24}$ Cells were cross-linked with formaldehyde, and the subsequent steps were performed 
according to the protocol of the ChIP kit (EZ-CHIP; Millipore, Burlington, MA). The ChIP primers are listed in Table 1.

\section{Constructs}

$M B D 2$ and TET1 overexpression plasmids were purchased from Vigene Bioscience (Jinan, China). The lentivirus used to stably overexpress or knock down MBD2 in the LUAD cell lines was purchased from Genechem (Shanghai, China).

\section{Western Blot Analysis and IHC}

Western blot and immunohistochemistry (IHC) analyses were performed, as previously described. ${ }^{22}$ Primary antibodies against MBD2, TET1, TET2, TET3, E-cadherin, N-cadherin, vimentin, fibronectin, ZEB1, and ZEB2 were purchased from GeneTex (Irvine, CA). Glyceraldehyde-3-phosphate dehydrogenase and horseradish peroxidase-conjugated secondary antibodies were purchased from Cell Signaling Technology (Danvers, MA). The IHC staining intensity of MBD2 was scored as follows: 0 (negative), 1 (weak), 2 (moderate), and 3 (strong). Negative and weak staining was defined as MBD2 low expression. Moderate and strong staining was defined as MBD2 high expression.

\section{Co-Immunoprecipitation}

The cell line was cultured in 6-well plates. Then, cells were harvested, and the co-immunoprecipitation was performed, as previously described. ${ }^{22}$
Animal Model

Six-week-old male BALB/c nude mice were used for the animal model. Mice were purchased from Shanghai SLAC Laboratory Animal Co, Ltd (Shanghai, China) and kept in specific pathogen-free conditions. Thirty mice were divided into three groups: MBD2, MBD2-( $\Delta 245-345)$, and negative control. Each group contains 10 mice. The wild-type MBD2, MBD2-( $\Delta 245-345)$, and empty vectoroverexpressing cell line HCC827 were subcutaneously injected into the mice at a concentration of $2 \times 10^{6} / 100 \mu \mathrm{L}$ per mouse in each group. All mice were euthanized at the end of the experiment. The tumor grafts from the mice were fixed for IHC analysis. Tumors were measured, and tumor volume was calculated, as previously described. ${ }^{22}$ The animal experiments were performed according to the Guide for the Care and Use of Laboratory Animals ${ }^{25}$ of Zhejiang Provincial People's Hospital.

\section{Statistical Analysis}

SPSS software version 19.0 (SPSS Inc., Chicago, IL) was used for statistical analysis. The Pearson correlation coefficient and the $\chi^{2}$ test were used to analyze the relationship between gene expression and the clinicopathological parameters. Other experimental data were analyzed via $t$-test. The overall survival was analyzed using the method of Kaplan-Meier and compared with the log-rank test. Values were considered statistically significant when $P<0.05$.

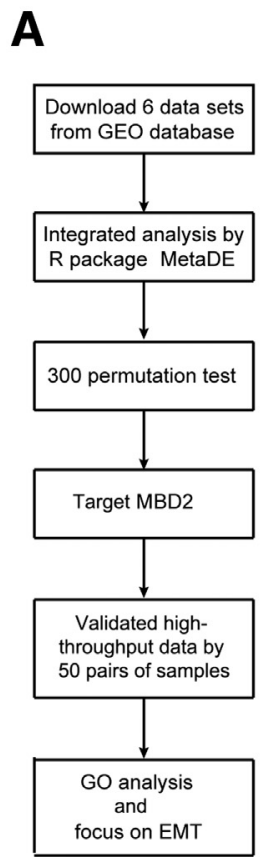

B

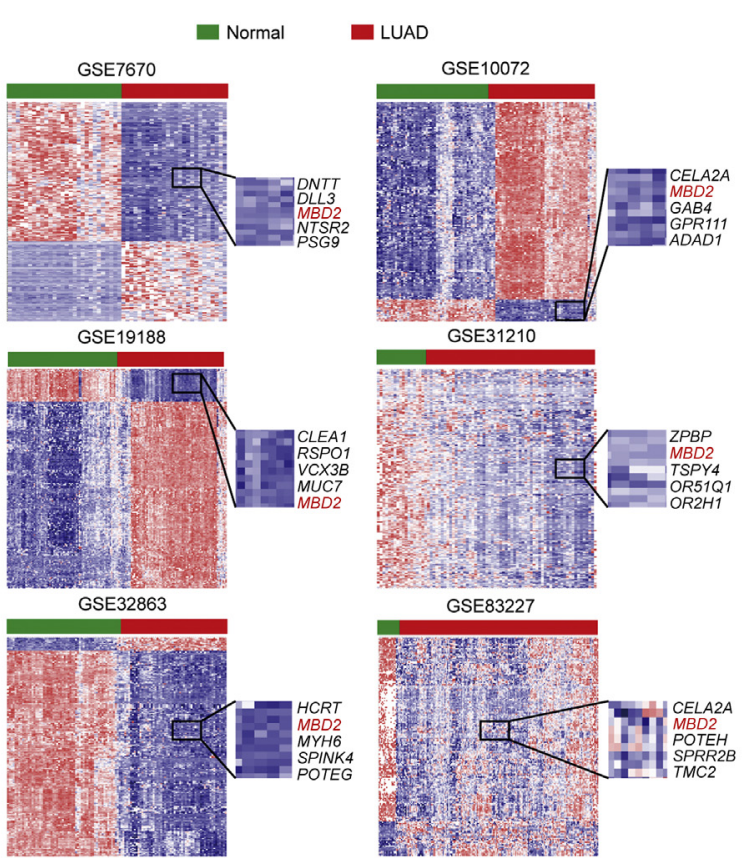

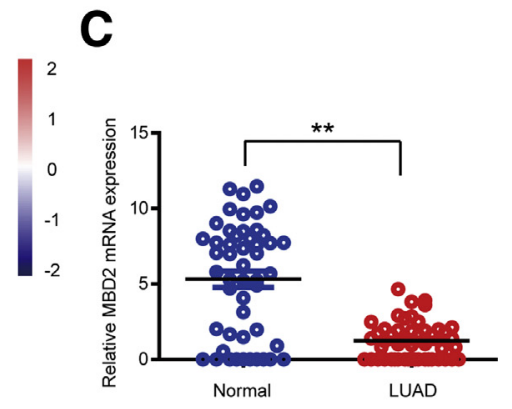

D

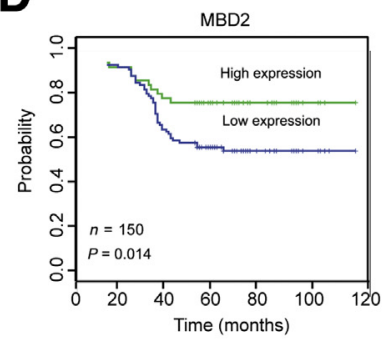

Figure 1 MBD2 is down-regulated in lung adenocarcinoma (LUAD) and associated with prognosis. A: Flowchart of the bioinformatic analysis of six Gene Expression Omnibus (GEO) data sets, followed by their validation. B: The down-regulation of MBD2 in LUAD tissues compared with nontumor tissues in six GEO data sets. C: MBD2 expression was validated in 50 pairs of tumor and nontumor samples. D: The Kaplan-Meier analysis of MBD2 expression in LUAD tissue-array. $n=150$ (D). ${ }^{* *} P<0.01$. EMT, epithelial-mesenchymal transition; G0, Gene Ontology. 
Table 2 The Correlation between MBD2 Expression Levels and Clinicopathological Data

\begin{tabular}{|c|c|c|c|c|}
\hline \multirow[b]{2}{*}{ Parameters } & \multicolumn{2}{|l|}{ MBD2 } & \multicolumn{2}{|c|}{$\chi^{2}$ Test value } \\
\hline & $\begin{array}{l}\text { Low } \\
(n=100)\end{array}$ & $\begin{array}{l}\text { High } \\
(n=50)\end{array}$ & & $P$ value \\
\hline \multicolumn{5}{|l|}{ Sex } \\
\hline Male & 28 & 7 & 3.652 & 0.056 \\
\hline Female & 72 & 43 & & \\
\hline \multicolumn{5}{|l|}{ Age, years } \\
\hline$<50$ & 53 & 21 & 1.614 & 0.204 \\
\hline$>50$ & 47 & 29 & & \\
\hline \multicolumn{5}{|l|}{ Smoking } \\
\hline Never smoker & 29 & 18 & 0.759 & 0.384 \\
\hline $\begin{array}{l}\text { Former or } \\
\text { current smoker }\end{array}$ & 71 & 32 & & \\
\hline \multicolumn{5}{|l|}{ Size, $\mathrm{cm}$} \\
\hline$<3$ & 56 & 32 & 0.880 & 0.348 \\
\hline$>3$ & 44 & 18 & & \\
\hline \multicolumn{5}{|l|}{ Stage } \\
\hline I/II & 42 & 33 & 7.680 & $0.006^{*}$ \\
\hline III/IV & 58 & 17 & & \\
\hline \multicolumn{5}{|l|}{ LN metastasis } \\
\hline Negative & 30 & 29 & 10.952 & $0.001^{*}$ \\
\hline Positive & 70 & 21 & & \\
\hline
\end{tabular}

GEO data sets were analyzed using R software version 3.4.2 (https://www.r-project.org). Differently expressed genes were filtered by $\mathrm{R}$ packages limma, MetaDE, and edgeR.

\section{Results}

MBD2 Is Down-Regulated in LUAD and Associated with Poor Survival

Six LUAD data sets were obtained from the GEO database (https://www.ncbi.nlm.nih.gov/geo; accession numbers GSE7670, GSE10072, GSE19188, GSE31210, GSE32863, and GSE8322), and integrated analyses were performed using the $\mathrm{R}$ package MetaDE (Figure 1A). Through 300 permutation tests, 205 differentially expressed mRNAs were identified (Supplemental Table S1). Among these differentially expressed mRNAs, $M B D 2$, which acts as an epigenetic modulator, was shown to be down-regulated in LUAD (Figure 1B). To explore the influence of epigenetic modulation in the LUAD biological process, the following study focused on MBD2.

To validate the down-regulation of MBD2 in the GEO data sets, $M B D 2$ expression was analyzed with qPCR in 50 pairs of tumor tissues and adjacent nontumor tissues that were obtained from LUAD patients. MBD2 was significantly down-regulated in tumor tissues compared with nontumor tissues (Figure 1C). Kaplan-Meier analysis of
LUAD tissue-array samples also revealed that a low expression of MBD2 indicated poorer survival in LUAD patients (Figure 1D). Analysis of the clinicopathological parameters revealed that MBD2 low expression was significantly correlated with higher tumor stage and lymph node metastasis (Table 2).

\section{MBD2 Inhibits Cell Migration}

To exclude sample differences, differences resulting from the microarray technology, and other biological confounding factors, forest plots of MBD2 expression in the GEO data sets were established. The summarization of the forest plots revealed the down-regulation of MBD2 in six independent data sets by integrated analysis (Figure 2A). Next, all LUAD samples in these six data sets were divided into the following two groups by MBD2 expression median: the MBD2 low-expression (MBD2low) group and the MBD2 high-expression (MBD2-high) group. Differentially expressed genes were identified by the $\mathrm{R}$ package edgeR. The differentially expressed genes in these two groups were subjected to a Gene Ontology analysis. Differentially expressed genes between the MBD2-high and MBD2-low groups were significantly correlated with cell adhesion and cell junction (Figure 2B). Furthermore, based on the correlation between MBD2 and metastasis (Table 2), it was hypothesized that MBD2 may influence cell migration and tumor metastasis in LUAD.

To validate this hypothesis, a cell migration assay was performed. First, $M B D 2$ mRNA levels were detected in various LUAD cell lines. H1975 and A549 exhibited the highest endogenous MBD2 expression, whereas the expression level of MBD2 was low in HCC827 and PC9 (Figure 2C). Furthermore, a lentivirus was used to overexpress MBD2 in HCC827 and PC9. MBD2 was also knocked down in H1975 and A549 using the lentivirus. Cell migration was inhibited by MBD2 overexpression in $\mathrm{HCC} 827$ and PC9, whereas the knockdown of MBD2 enhanced cell migration in H1975 and A549 (Figure 2D). Collectively, these results demonstrate that MBD2 acted as a tumor suppressor by inhibiting cell migration.

\section{MBD2 Suppresses Tumor Metastasis by Up-Regulating miR-200s}

The mechanism through which MBD2 suppresses cell migration was investigated. Given that the EMT process is profoundly involved in cell migration and metastasis, Western blot analysis was used to detect alterations in EMT markers. The overexpression of MBD2 inhibited the EMT process, whereas the opposite result was obtained with the knockdown of MBD2 (Figure 3A). Next, the mechanism through which MBD2 suppresses the EMT process was investigated. Multiple miRNAs suppress tumor metastasis, such as the miR-126, miR-148a, miR-152, and miR-200 families. Therefore, the expression of these miRNAs, which suppress invasive properties of tumors, was detected by qPCR. MBD2 had no effect on most of these miRNAs, 

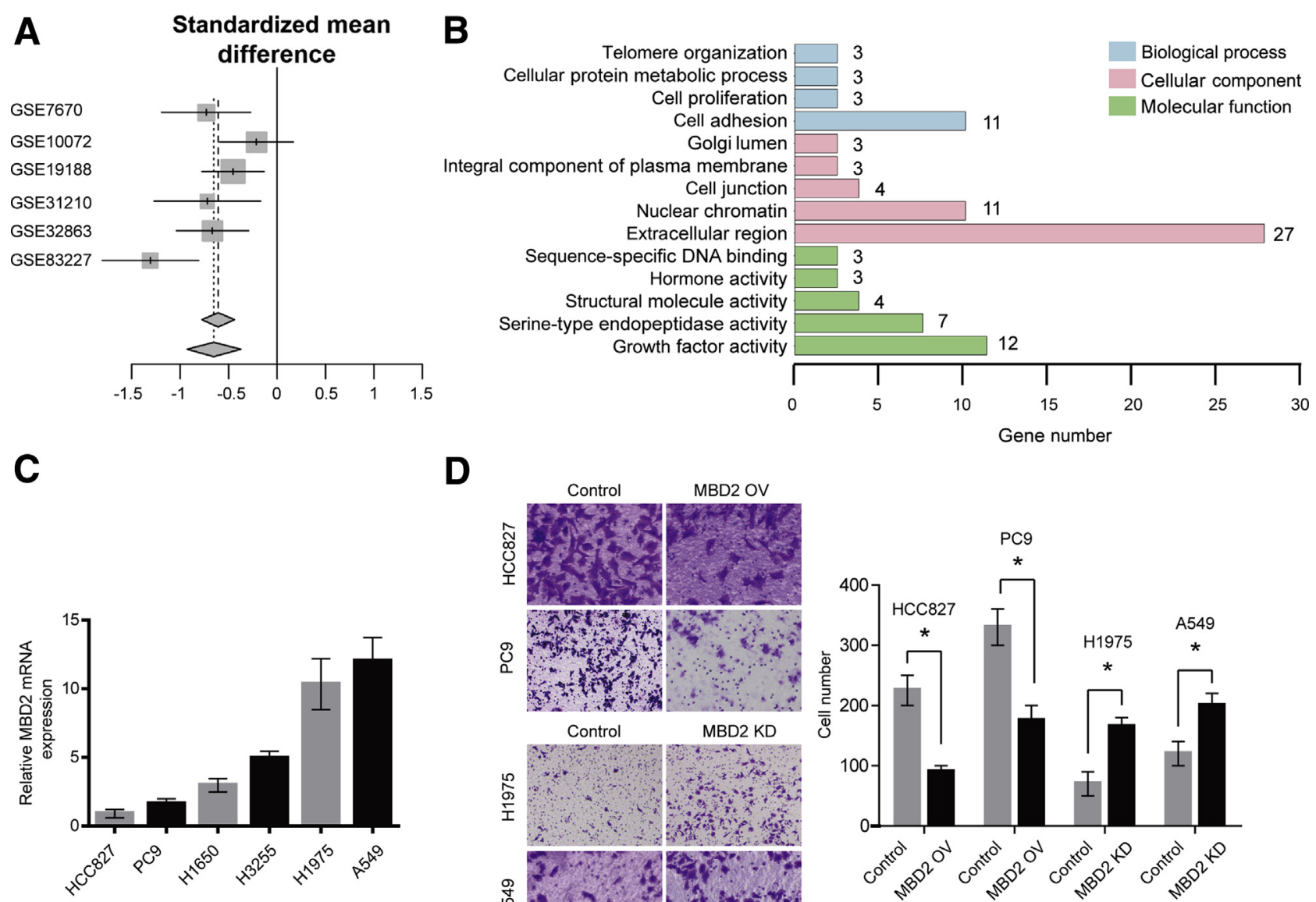

D

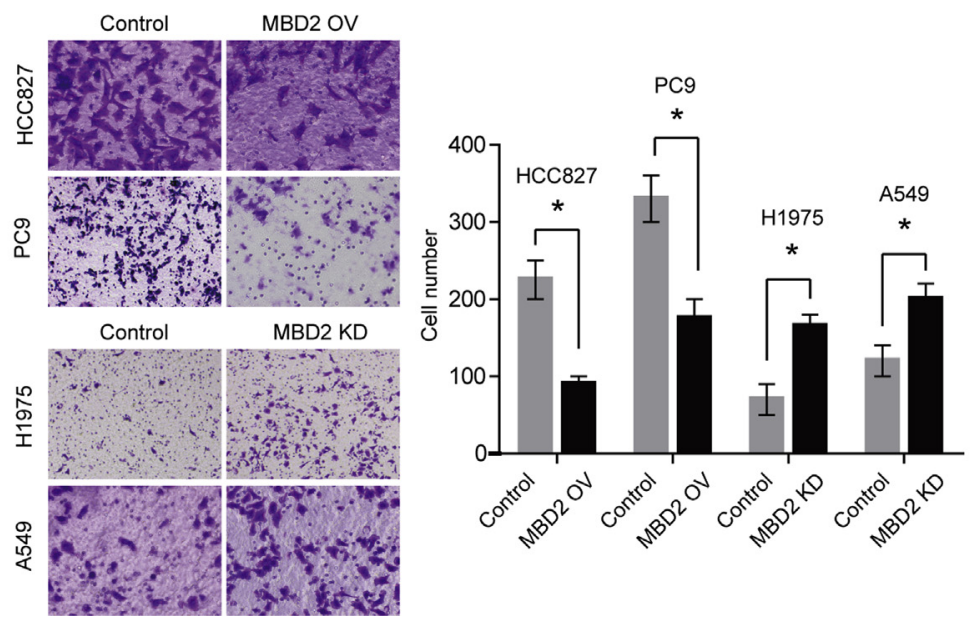

Figure 2 MBD2 inhibits cell migration. A: The forest plots summarize MBD2 expression in six Gene Expression Omnibus data sets. B: The Gene Ontology analysis of differentially expressed genes between the MBD2-high and MBD2-low groups. These differently expressed genes were enriched by biological process, cellular component, and molecular function with $P<0.05$. C: MBD2 expression in lung adenocarcinoma cell lines (HCC827, PC9, H1650, H3255, H1975, and A549). D: MBD2 overexpression in HCC827 and PC9 inhibits cell migration. MBD2 knockdown in H1975 and A549 promotes cell migration. Data are expressed as means \pm SD (C and $\mathbf{D})$. ${ }^{*} P<0.05 . K D$, knockdown; OV, overexpression.

except for the miR-200 family (Figure 3, B and C). Further analysis of MBD2 and the miR-200s in clinical LUAD samples revealed a significant positive correlation between MBD2 and the miR-200s (Figure 3D). It has been reported that the miR-200s suppress the cellular EMT by targeting ZEB1 and ZEB2 in various types of tumors, such as LUAD, colon rectal cancer, and prostate cancer. ${ }^{26}$ As a result, it was concluded that MBD2 positively correlates with miR-200s and may suppress cell migration and tumor metastasis by up-regulating the miR-200s in LUAD cells.

\section{MBD2 Correlates with TET1 and 5-hmC Content}

Next, the mechanism underlying the regulation of the miR200s by MBD2 was investigated. Wang et $\mathrm{al}^{27}$ reported that MBD2 promotes the demethylation of Foxp3 promoter through TET1 in regulatory $\mathrm{T}$ cells. In that study, the depletion of MBD2 induced an obvious down-regulation of TET1. We hypothesized that a similar mechanism may account for the correlation between MBD2 and TET1 in
LUAD. The overexpression of MBD2 elevated TET1 levels, whereas the knockdown of MBD2 down-regulated TET1 in the cell lines (Figure 4A). Furthermore, qPCR analysis of the clinical samples also revealed a positive correlation between MBD2 and TET1 (Figure 4B). In addition, LUAD patients with high expression levels of TET1 had a better prognosis (Figure 4C).

TET1 activates gene expression by converting 5-mC to 5$\mathrm{hmC}$ and promoting the demethylation of CGIs. Therefore, it was explored whether MBD2 regulates the content of 5$\mathrm{mC}$ and 5-hmC in the promoter of miR-200s. Clinical samples were subjected to methylation-sensitive restriction digestion, followed by qPCR analysis. The results revealed that samples with high MBD2 expression exhibited a lower 5 -mC content in the promoter of miR-200s (Figure 4D). Bisulfite-sequencing PCR confirmed these results that the MBD2-high group had fewer methylated CGIs in the promoter of miR-200s compared with the MBD2-low group (Figure 4E). The 5-hmC content in CGIs was detected by glucosylation of genomic DNA followed by methylation- 

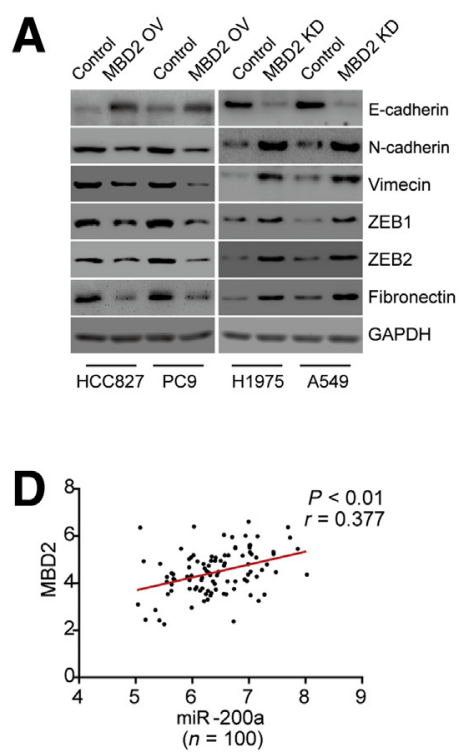
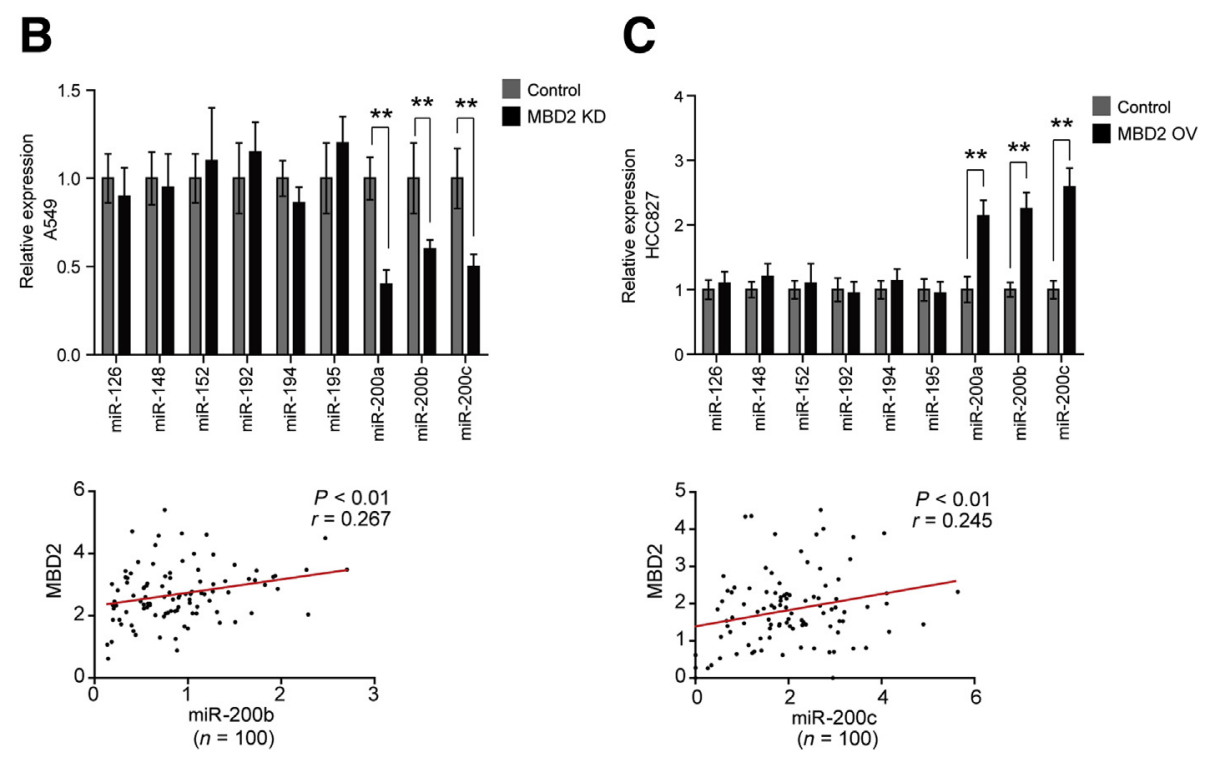

Figure 3 MBD2 suppresses tumor metastasis through miR-200s. A: The expression of epithelial-mesenchymal transition markers in the MBD2 overexpression cell lines (HCC827 and PC9) and MBD2 knockdown cell lines (H1975 and A549). B: The expression of miRNAs that suppress invasive properties in the MBD2 knockdown cell line A549. C: The expression of miRNAs that suppress invasive properties in the MBD2 overexpression cell line HCC827. D: The positive correlation between MBD2 and miR-200s in lung adenocarcinoma clinical samples (fit red lines with linear regression). $n=100$ (D). Data are expressed as means \pm SD (B and $\mathbf{C})$. ${ }^{*} P<0.01$. Ctrl, control; GAPDH, glyceraldehyde-3-phosphate dehydrogenase; KD, knockdown; OV, overexpression; ZEB, zinc finger Ebox binding homeobox.

sensitive-qPCR. The MBD2-high group exhibited a higher 5 -hmC content in the promoter of miR-200s compared with the MBD2-low group (Figure 4F). Furthermore, Pearson's correlation analysis of the clinical samples also revealed a positive correlation between TET1 and the miR-200s (Figure 4G). Collectively, these results indicate that MBD2 correlates with TET1 expression and 5-hmC content in the promoter of miR-200s.

\section{MBD2 Inhibits EMT by Interacting with TET1}

Wang et $\mathrm{al}^{27}$ hypothesized that MBD2 recruits TET1 to the gene promoter, but they did not provide direct evidence of this phenomenon. In the present study, immunoprecipitation was performed in LUAD cells, and the direct interaction between MBD2 and TET1 was observed (Figure 5A). To investigate whether the interaction between MBD2 and TET1 is necessary for the expression of the miR-200s and the EMT process, various truncations of MBD2 were constructed. The immunoprecipitation of these MBD2 truncations and TET1 revealed that amino acids 245 to 345 are critical for the interaction between MBD2 and TET1. Furthermore, MBD2-( $\Delta 245-345)$, which lacked amino acids 245 to 345 , failed to interact with TET1 (Figure 5B). The subsequent cell migration assay and Western blot assay confirmed that only wild-type MBD2 inhibited cell migration and the EMT process; this effect was not observed with the MBD2-( $\Delta 245-345)$ truncation, which lacked amino acids 245 to 345 (Figure 5, C and D). These results indicate that the interaction between MBD2 and TET1 is indispensable for the inhibition of EMT.

\section{MBD2 Recruits TET1 to CGIs of the TET1 Promoter}

The mechanism through which MBD2 regulates TET1 expression remains unclear. Sun et $\mathrm{al}^{21}$ reported that TET1 participates in autoregulation. In that study, a ChIP assay revealed that TET1 binds to its own promoter. Because there are 66 CGIs in the TET1 promoter, it was hypothesized that MBD2 regulates TET1 expression by recruiting TET1 to the TET1 promoter. To test this hypothesis, first, the content of 5-mC and 5-hmC was analyzed in the TETI promoter. Clinical samples with high levels of MBD2 contained more unmethylated CGIs and a higher 5-hmC content in the TET1 promoter when compared with samples with low MBD2 expression (Figure 6, A and B). Next, a ChIP assay was performed to obtain direct evidence that MBD2 binds to the TET1 promoter. The A549 cell line was chosen because this cell line exhibited the highest endogenous MBD2 expression. The enrichment of MBD2 to the TET1 promoter was observed by ChIP-PCR (Figure 6C). To confirm that MBD2 recruits TET1 to the TET1 promoter, the HCC827 cell line was chosen because this cell line exhibited almost no MBD2 expression. First, the HCC827/TET1 cell line was constructed, in which TET1 was stably expressed. Then, wild-type MBD2 or MBD2-( $\Delta 245-345)$ was overexpressed in the HCC827/TET1 cell line. The overexpression of wild-type MBD2 resulted in the recruitment of TET1 to the TET1 promoter, but this was not the case for MBD2-( $\Delta 245-345)$ (Figure 6D). These results indicate that amino acids 245 to 345 are critical for the recruitment of TET1 and MBD2 to the TET1 promoter. The following experiment, in which the content of 5$\mathrm{mC}$ and 5-hmC was analyzed in the cell lines, also demonstrated that the overexpression of wild-type MBD2, but not MBD2- 
A
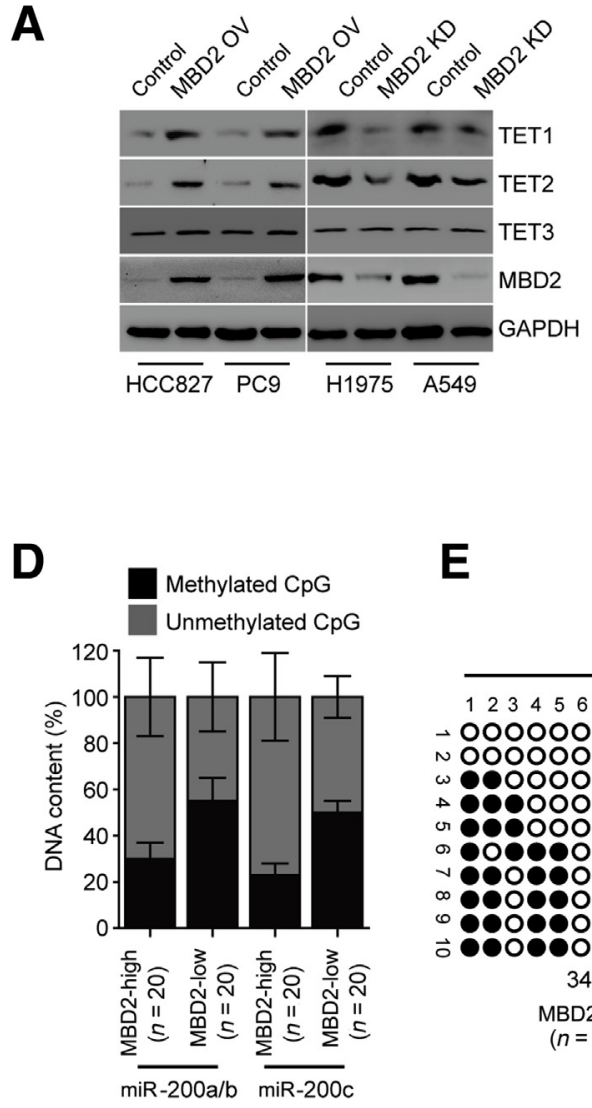

E
B

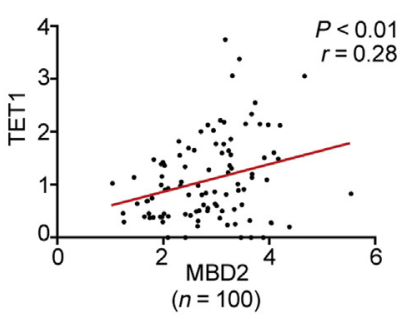

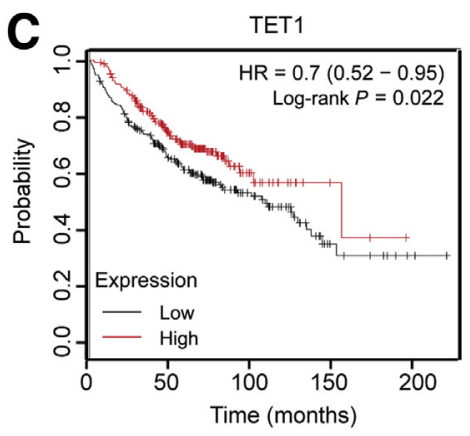

Number at risk

$\begin{array}{llllll}\text { Low } & 231 & 117 & 33 & 7 & 0 \\ \text { High } & 230 & 110 & 11 & 2 & 0\end{array}$

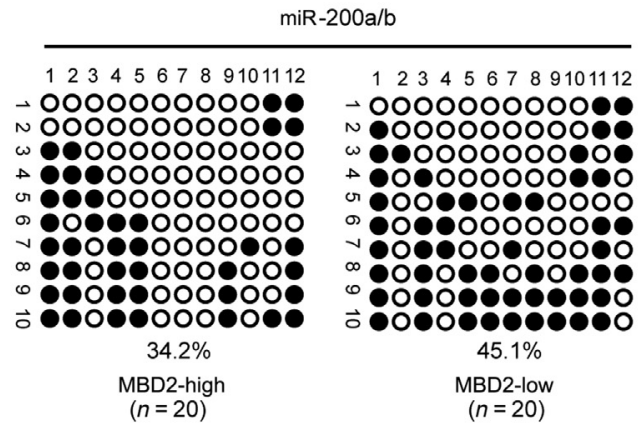

$\mathbf{G}$

$\mathbf{F}$
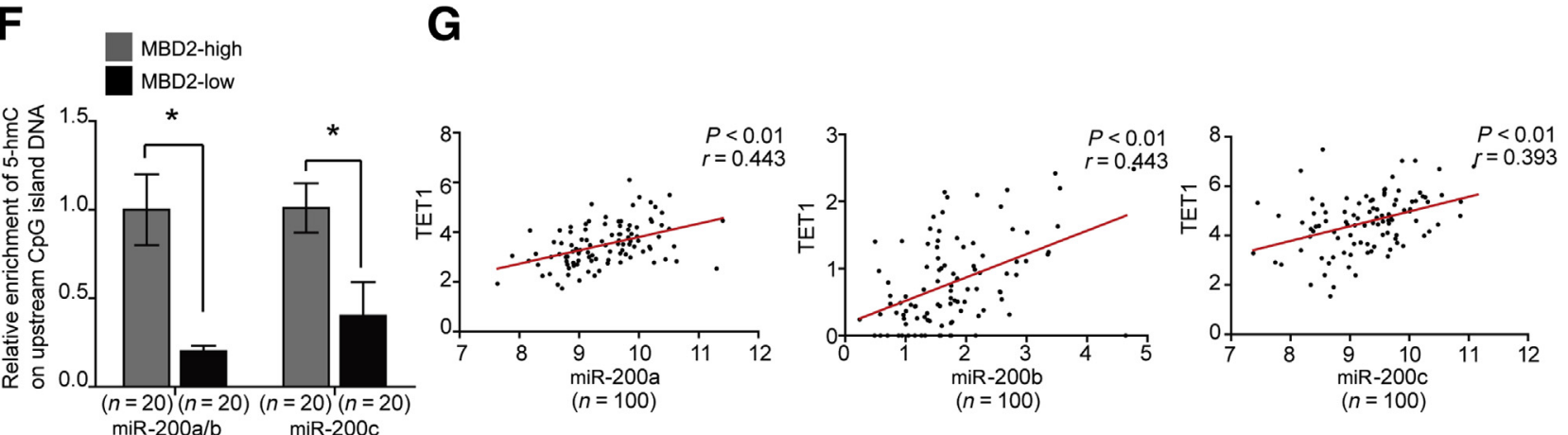

Figure 4 MBD2 correlates with TET1 and 5-hydroxymethylcytosine (5-hmC) content. A: TET1, TET2, and TET3 expression in the MBD2 overexpression cell lines (HCC827 and PC9) and MBD2 knockdown cell lines (H1975 and A549). B: The positive correlation between MBD2 and TET1 in lung adenocarcinoma (LUAD) clinical samples (fit red line with linear regression). C: The Kaplan-Meier analysis of TET1 expression in LUAD patients by the Kaplan-Meier Plotter online tool (http://www. kmplot.com, last accessed December 2, 2018). D: Methylated and unmethylated CpG islands (CGIs) in the MBD2-high and MBD2-low LUAD clinical samples. E: Bisulfitesequencing PCR of CGIs upstream of miR-200s in MBD2-high and MBD2-low LUAD clinical samples. F: The 5-hmC content upstream of miR-200s in the MBD2-high and MBD2-low LUAD clinical samples. G: The positive correlation between TET1 and miR-200s in LUAD clinical samples (fit red lines with linear regression). $n=100$ (B and $\mathbf{G}) ; n=20$ (D-F, MBD2-high and MBD2-low samples). Data are expressed as means \pm SD (D and F). ${ }^{*} P<0.05$. Ctrl, control; GAPDH, glyceraldehyde-3-phosphate dehydrogenase; $\mathrm{HR}$, hazard ratio; KD, knockdown; OV, overexpression.

( $\Delta 245-345)$, increased the unmethylated CGIs and the 5-hmC content in the TET1 promoter (Figure 6, E and F).

\section{MBD2 Inhibits Tumor Growth in Vivo}

An animal model was used to investigate the antioncogenic effect of MBD2 in vivo. Wild-type MBD2 significantly reduced the tumor volume and tumor weight (Figure $6 \mathrm{G})$. In contrast, MBD2-( $\Delta 245-345)$ had no effect on tumor growth in the xenograft model. IHC analysis confirmed that wildtype MBD2, but not MBD2-( $\Delta 245-345)$, increased TET1 expression in the animal model (Figure $6 \mathrm{H}$ ).

\section{Discussion}

Earlier studies demonstrated that MBD2 always acts as a tumor activator in various types of carcinomas. MBD2 
A
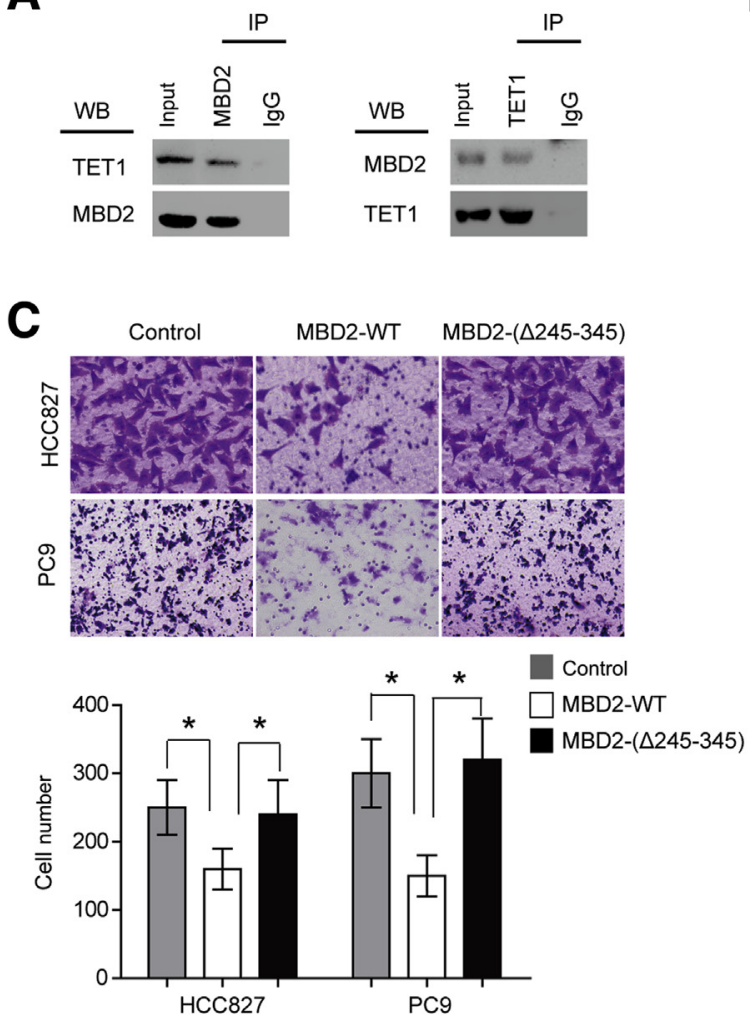

B

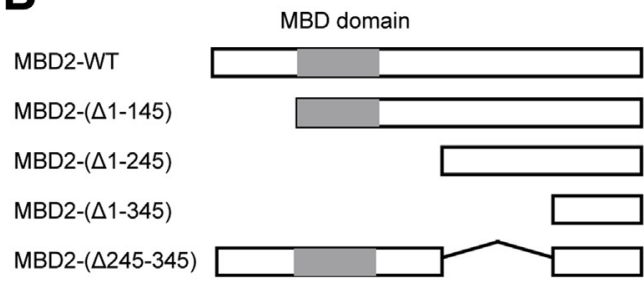

TET1-HA

MBD2-WT-flag

MBD2-( $\triangle 1-145)$-flag

MBD2-( $\triangle 1-245)$-flag

MBD2-( $\Delta 1-345)$-flag

MBD2-( $\Delta 245-345)-$ flag

IP: $\alpha$-flag
WB: $\alpha-H A$

Input

WB: $\alpha-H A$
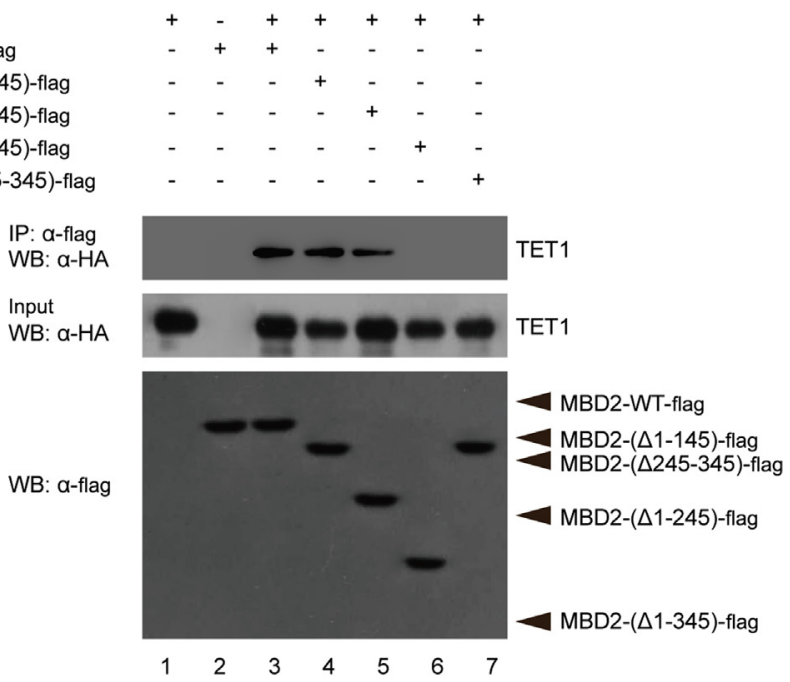

D

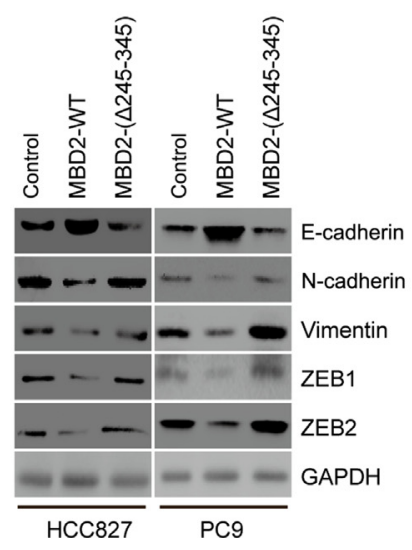

Figure 5 MBD2 inhibits epithelial-mesenchymal transition (EMT) by interacting with TET1. A: The co-immunoprecipitation of MBD2 and TET1 in lung adenocarcinoma (LUAD) cell line A549. B: The co-immunoprecipitation of various MBD2 truncations and TET1. C: Overexpression of wild-type (WT) MBD2, but not MBD2-( $\Delta 245-345)$, inhibits cell migration in LUAD cell lines HCC827 and PC9. D: Overexpression of wild-type MBD2, but not MBD2-( $\Delta 245-345)$, inhibits the EMT process in LUAD cell lines HCC827 and PC9. Data are expressed as means \pm SD (C). ${ }^{*} P<0.05$. Ctrl, control; GAPDH, glyceraldehyde-3phosphate dehydrogenase; HA, hemagglutinin; IP, immunoprecipitation; WB, Western blot analysis; ZEB, zinc finger E-box binding homeobox.

proteins form complexes by recruiting other proteins, such as DNA methyltransferases (DNMTs), HDACs, and SIN3 transcription regulator family member A (SIN3A), which are all transcriptional corepressors. ${ }^{28,29}$ By binding to the promoter or enhancer regions of specific tumor-suppressor genes that are mediated by MBD2, these complexes induce a condensed and inactive chromosomal structure. Compared with the role of MBD2 in other malignant tumors, research on the role of MBD2 in LUAD progression remains scarce. Contrary to the conventional view that MBD2 acts as a tumor activator, the present study demonstrated that MBD2 was down-regulated in LUAD through the bioinformatics analysis of the GEO data sets and validation with clinical samples. In addition, it was determined that depressed expression of MBD2 indicates poor survival. Further experiments in cell lines and animal models also revealed that MBD2 inhibits metastasis. These findings reveal an antitumor function of MBD2 in LUAD. MBD2 exhibits cellular- and tissue-specific functions in a context-dependent manner, which may explain the contradiction between the antitumor function of MBD2 observed in the present study and its protumor action in other reports.

To date, miRNA has been widely studied in the progression of malignant tumors. Aberrantly expressed miRNAs are strongly involved in carcinogenesis. It has been reported that certain miRNAs participate in epigenetic modulation by targeting DNA methyltransferases (DNMT1 and DNMT3b). ${ }^{30-32}$ However, not many studies have investigated whether miRNAs can be epigenetically regulated. Chuang et $\mathrm{al}^{20}$ reported the regulation of miRNAs by 

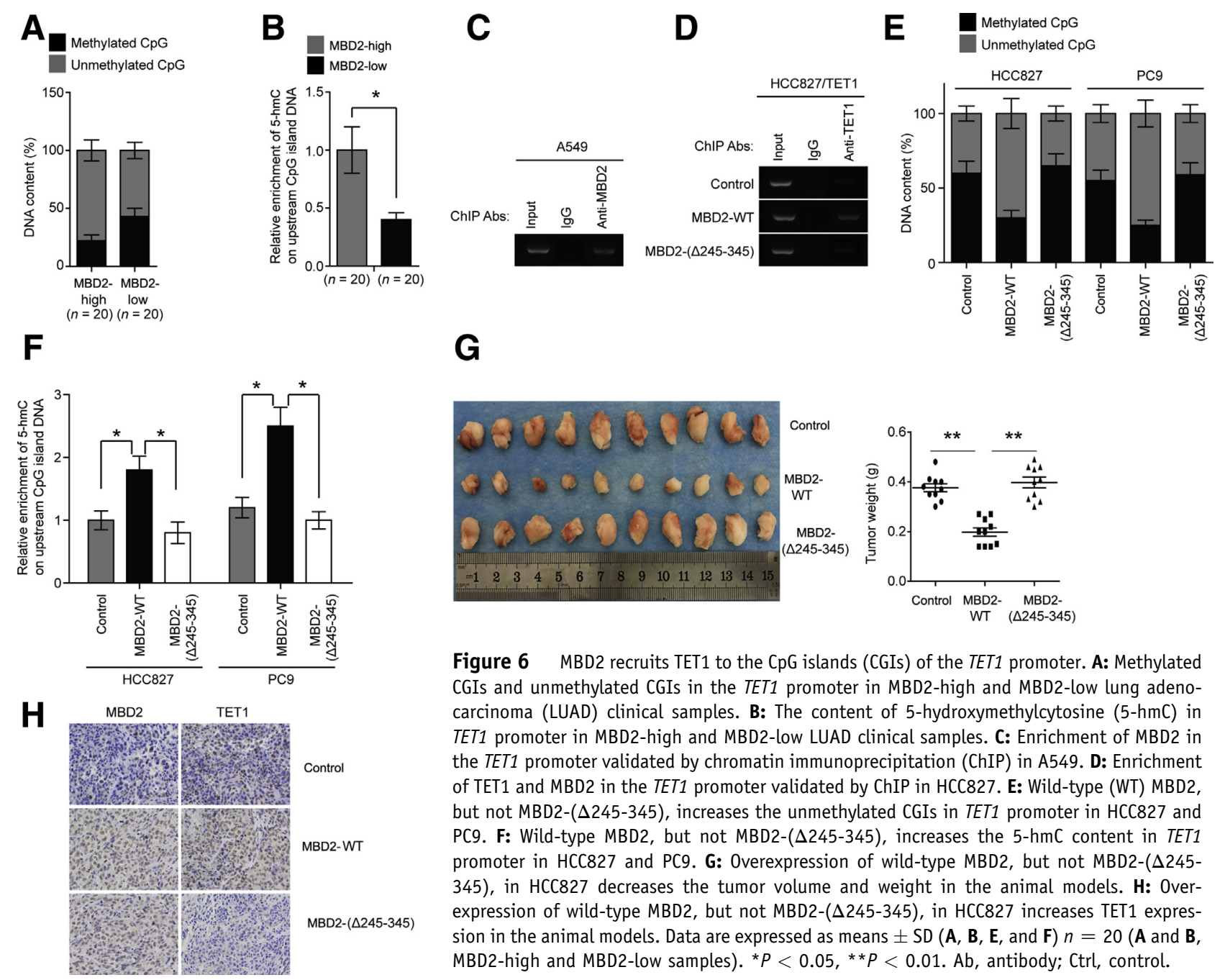

Figure 6 MBD2 recruits TET1 to the CpG islands (CGIs) of the TET1 promoter. A: Methylated CGIs and unmethylated CGIs in the TET1 promoter in MBD2-high and MBD2-low lung adenocarcinoma (LUAD) clinical samples. B: The content of 5-hydroxymethylcytosine (5-hmC) in TET1 promoter in MBD2-high and MBD2-low LUAD clinical samples. C: Enrichment of MBD2 in the TET1 promoter validated by chromatin immunoprecipitation (ChIP) in A549. D: Enrichment of TET1 and MBD2 in the TET1 promoter validated by ChIP in HCC827. E: Wild-type (WT) MBD2, but not MBD2-( $\triangle 245-345)$, increases the unmethylated CGIs in TET1 promoter in HCC827 and PC9. F: Wild-type MBD2, but not MBD2-( $\Delta 245-345)$, increases the 5-hmC content in TET1 promoter in HCC827 and PC9. G: Overexpression of wild-type MBD2, but not MBD2-( $\Delta 245-$ $345)$, in HCC827 decreases the tumor volume and weight in the animal models. H: Overexpression of wild-type MBD2, but not MBD2-( $\Delta 245-345)$, in HCC827 increases TET1 expression in the animal models. Data are expressed as means $\pm \mathrm{SD}(\mathbf{A}, \mathbf{B}, \mathbf{E}$, and $\mathbf{F}) n=20$ (A and $\mathbf{B}$, MBD2-high and MBD2-low samples). ${ }^{*} P<0.05,{ }^{*} P<0.01$. Ab, antibody; Ctrl, control.

5-mC and 5-hmC upstream. In the present study, it was demonstrated that the EMT-suppressing miR-200s may be regulated by a similar mechanism. The expression of MBD2 was positively correlated with the expression of miR-200s and the content of $5-\mathrm{hmC}$ in the promoter of miR-200s in clinical samples. It has been reported that 5 -hmC induces hypomethylation in the gene promoter. And it was also observed that hypomethylation status in the promoter of miR-200s occurred in MBD2-high samples, whereas MBD2-low samples had hypermethylation status in the promoter of miR-200s. Although the miR-200s correlated with 5-hmC content in the present study, acetylation of H3K27 (H3K27Ac) histone enrichment sites were also observed upstream of the miR-200s. ${ }^{33}$ The H3K27Ac histone mark, which is the acetylation of lysine 27 of the H3 histone, has been considered as a marker of transcriptional activation. However, it remains unclear whether the histone acetylation that occurs in the promoter of miR-200s makes sense in the malignant transformation of LUAD. This question warrants further investigation.
TET1 is a member of the 10 to 11 translocation family that regulates specific gene expression by modulating the 5$\mathrm{hmC}$ content in the promoter. Previous studies have shown that the loss of the TET family and 5-hmC are common during the progression of solid tumors, including LUAD. ${ }^{18}$ TET1 expression is regulated by many factors. Sun et $\mathrm{al}^{21}$ demonstrated that the down-regulation of TET1 in breast cancer is caused by the hypermethylation status in its promoter. Chuang et $\mathrm{al}^{20}$ reported that TET1 is regulated by miR-494. In the present study, it was determined that MBD2 recruits TET1 and forms a complex, which locates to the TETI promoter and activates its transcription. However, there were also limitations inherent to the present study. It has been reported that MBD2 interacts with HDAC1, HDAC2, DNMT1, and SIN3A. ${ }^{28,29}$ These proteins always act as transcriptional suppressors by inducing a condensed and inactive chromosomal structure. It remains unknown whether MBD2 recruits these proteins and suppresses a specific oncogene or antioncogene in LUAD. Hence, further investigations are warranted. 
Overall, in the present study, the tumor-suppressive effect of MBD2 in LUAD was revealed. The antitumor effect of MBD2 is mediated by miR-200s and TET1. We also demonstrated the positive correlation between MBD2, miR200s, and TET1 in LUAD.

\section{Acknowledgment}

We thank Dr. Hong-ying Duan for editing and comments.

\section{Supplemental Data}

Supplemental material for this article can be found at https://doi.org/10.1016/j.ajpath.2019.01.010.

\section{References}

1. Lee YS, Bae SC: How do K-RAS-activated cells evade cellular defense mechanisms? Oncogene 2016, 35:827-832

2. Sivakumar S, Lucas FAS, McDowell TL, Lang W, Xu L, Fujimoto J, Zhang J, Futreal PA, Fukuoka J, Yatabe Y, Dubinett SM, Spira AE, Fowler J, Hawk ET, Wistuba II, Scheet P, Kadara H: Genomic landscape of atypical adenomatous hyperplasia reveals divergent modes to lung adenocarcinoma. Cancer Res 2017, 77:6119-6130

3. Li Z, Hou P, Fan D, Dong M, Ma M, Li H, Yao R, Li Y, Wang G, Geng P, Mihretab A, Liu D, Zhang Y, Huang B, Lu J: The degradation of EZH2 mediated by lncRNA ANCR attenuated the invasion and metastasis of breast cancer. Cell Death Differ 2017, 24:59-71

4. Cramer JM, Scarsdale JN, Walavalkar NM, Buchwald WA, Ginder GD, Williams DC Jr: Probing the dynamic distribution of bound states for methylcytosine-binding domains on DNA. J Biol Chem 2014, 289:1294-1302

5. Tatematsu KI, Yamazaki T, Ishikawa F: MBD2-MBD3 complex binds to hemi-methylated DNA and forms a complex containing DNMT1 at the replication foci in late S phase. Genes Cells 2000, 5:677-688

6. Humphrey GW, Wang Y, Russanova VR, Hirai T, Qin J, Nakatani Y, Howard BH: Stable histone deacetylase complexes distinguished by the presence of SANT domain proteins CoREST/kiaa0071 and MtaL1. J Biol Chem 2001, 276:6817-6824

7. Fujita H, Fujii R, Aratani S, Amano T, Fukamizu A, Nakajima T: Antithetic effects of MBD2a on gene regulation. Mol Cell Biol 2003, 23:2645-2657

8. Stefanska B, Suderman M, Machnes Z, Bhattacharyya B, Hallett M, Szyf M: Transcription onset of genes critical in liver carcinogenesis is epigenetically regulated by methylated DNA-binding protein MBD2. Carcinogenesis 2013, 34:2738-2749

9. Devailly G, Grandin M, Perriaud L, Mathot P, Delcros JG, Bidet Y, Morel AP, Bignon JY, Puisieux A, Mehlen P, Dante R: Dynamics of MBD2 deposition across methylated DNA regions during malignant transformation of human mammary epithelial cells. Nucleic Acids Res 2015, 43:5838-5854

10. Yuan K, Xie K, Fox J, Zeng H, Gao H, Huang C, Wu M: Decreased levels of miR-224 and the passenger strand of miR-221 increase MBD2, suppressing maspin and promoting colorectal tumor growth and metastasis in mice. Gastroenterology 2013, 145:853-864.e9

11. Bahrami A, Aledavood A, Anvari K, Hassanian SM, Maftouh M, Yaghobzade A, Salarzaee O, ShahidSales S, Avan A: The prognostic and therapeutic application of microRNAs in breast cancer: tissue and circulating microRNAs. J Cell Physiol 2018, 233:774-786

12. Abisoye-Ogunniyan A, Lin $\mathrm{H}$, Ghebremedhin A, Salam AB, Karanam B, Theodore S, Jones-Trich J, Davis M, Grizzle W, Wang H, Yates C: Transcriptional repressor Kaiso promotes epithelial to mesenchymal transition and metastasis in prostate cancer through direct regulation of miR-200c. Cancer Lett 2018, 431:1-10

13. Shelton PM, Duran A, Nakanishi Y, Reina-Campos M, Kasashima H, Llado V, Ma L, Campos A, Garcia-Olmo D, Garcia-Arranz M, GarciaOlmo DC, Olmedillas-Lopez S, Caceres JF, Diaz-Meco MT, Moscat J: The secretion of miR-200s by a PKCzeta/ADAR2 signaling axis promotes liver metastasis in colorectal cancer. Cell Rep 2018, 23: $1178-1191$

14. Tahiliani M, Koh KP, Shen Y, Pastor WA, Bandukwala H, Brudno Y, Agarwal S, Iyer LM, Liu DR, Aravind L, Rao A: Conversion of 5methylcytosine to 5-hydroxymethylcytosine in mammalian DNA by MLL partner TET1. Science 2009, 324:930-935

15. Guo JU, Su Y, Zhong C, Ming GL, Song H: Hydroxylation of 5methylcytosine by TET1 promotes active DNA demethylation in the adult brain. Cell 2011, 145:423-434

16. Chen Q, Yin D, Zhang Y, Yu L, Li XD, Zhou ZJ, Zhou SL, Gao DM, Hu J, Jin C, Wang Z, Shi YH, Cao Y, Fan J, Dai Z, Zhou J: MicroRNA-29a induces loss of 5-hydroxymethylcytosine and promotes metastasis of hepatocellular carcinoma through a TET-SOCS1-MMP9 signaling axis. Cell Death Dis 2017, 8:e2906

17. Neri F, Dettori D, Incarnato D, Krepelova A, Rapelli S, Maldotti M, Parlato C, Paliogiannis P, Oliviero S: TET1 is a tumour suppressor that inhibits colon cancer growth by derepressing inhibitors of the WNT pathway. Oncogene 2015, 34:4168-4176

18. Forloni M, Gupta R, Nagarajan A, Sun LS, Dong Y, Pirazzoli V, Toki M, Wurtz A, Melnick MA, Kobayashi S, Homer RJ, Rimm DL, Gettinger SJ, Politi K, Dogra SK, Wajapeyee N: Oncogenic EGFR represses the TET1 DNA demethylase to induce silencing of tumor suppressors in cancer cells. Cell Rep 2016, 16:457-471

19. Tao QF, Yuan SX, Yang F, Yang S, Yang Y, Yuan JH, Wang ZG, Xu QG, Lin KY, Cai J, Yu J, Huang WL, Teng XL, Zhou CC, Wang F, Sun SH, Zhou WP: Aldolase B inhibits metastasis through Ten-Eleven Translocation 1 and serves as a prognostic biomarker in hepatocellular carcinoma. Mol Cancer 2015, 14:170

20. Chuang KH, Whitney-Miller CL, Chu CY, Zhou Z, Dokus MK, Schmit S, Barry CT: MicroRNA-494 is a master epigenetic regulator of multiple invasion-suppressor microRNAs by targeting ten eleven translocation 1 in invasive human hepatocellular carcinoma tumors. Hepatology 2015, 62:466-480

21. Sun M, Song CX, Huang H, Frankenberger CA, Sankarasharma D, Gomes S, Chen P, Chen J, Chada KK, He C, Rosner MR: HMGA2/TET1/HOXA9 signaling pathway regulates breast cancer growth and metastasis. Proc Natl Acad Sci U S A 2013, 110: 9920-9925

22. Pei YF, Yin XM, Liu XQ: TOP2A induces malignant character of pancreatic cancer through activating beta-catenin signaling pathway. Biochim Biophys Acta Mol Basis Dis 2018, 1864:197-207

23. Pei YF, Lei Y, Liu XQ: MiR-29a promotes cell proliferation and EMT in breast cancer by targeting ten eleven translocation 1. Biochim Biophys Acta 2016, 1862:2177-2185

24. Pei YF, Tao R, Li JF, Su LP, Yu BQ, Wu XY, Yan M, Gu QL, Zhu ZG, Liu BY: TET1 inhibits gastric cancer growth and metastasis by PTEN demethylation and re-expression. Oncotarget 2016, 7: 31322-31335

25. Committee for the Update of the Guide for the Care and Use of Laboratory Animals; National Research Council: Guide for the Care and Use of Laboratory Animals: Eighth Edition. Washington, DC, National Academies Press, 2011

26. Shu C, Yan D, Mo Y, Gu J, Shah N, He J: Long noncoding RNA IncARSR promotes epithelial ovarian cancer cell proliferation and invasion by association with HuR and miR-200 family. Am J Cancer Res 2018, 8:981-992

27. Wang L, Liu Y, Han R, Beier UH, Thomas RM, Wells AD, Hancock WW: Mbd2 promotes foxp3 demethylation and T-regulatorycell function. Mol Cell Biol 2013, 33:4106-4115

28. Boeke J, Ammerpohl O, Kegel S, Moehren U, Renkawitz R: The minimal repression domain of MBD2b overlaps with the methyl-CpG- 
binding domain and binds directly to Sin3A. J Biol Chem 2000, 275 34963-34967

29. Ng HH, Zhang Y, Hendrich B, Johnson CA, Turner BM, ErdjumentBromage $\mathrm{H}$, Tempst $\mathrm{P}$, Reinberg D, Bird A: MBD2 is a transcriptional repressor belonging to the MeCP1 histone deacetylase complex. Nat Genet 1999, 23:58-61

30. Braconi C, Huang N, Patel T: MicroRNA-dependent regulation of DNA methyltransferase-1 and tumor suppressor gene expression by interleukin-6 in human malignant cholangiocytes. Hepatology 2010, $51: 881-890$
31. Huang J, Wang Y, Guo Y, Sun S: Down-regulated microRNA-152 induces aberrant DNA methylation in hepatitis B virus-related hepatocellular carcinoma by targeting DNA methyltransferase 1. Hepatology 2010, 52:60-70

32. Duursma AM, Kedde M, Schrier M, le Sage C, Agami R: miR-148 targets human DNMT3b protein coding region. RNA 2008, 14:872-877

33. Ning X, Shi Z, Liu X, Zhang A, Han L, Jiang K, Kang C, Zhang Q: DNMT1 and EZH2 mediated methylation silences the microRNA 200b/a/429 gene and promotes tumor progression. Cancer Lett 2015, 359:198-205 